the sample is moved by microfluidics through a glass capillary, where lasers excite the probes at different wavelengths. A target miRNA molecule is counted when photons of both colours are emitted simultaneously.

Both conventional microarrays and beadbased multiplex assay platforms such as XMAP from Luminex of Austin, Texas, can be used to study miRNA expression, and a number of companies offer miRNA products designed for use with microarray systems. PerkinElmer of Boston, Massachusetts, sells a MICROMAX ASAP labeling kit for miRNAs for detection by the tyramide signal amplification (TSA) method, while the Array 900miRNA labeling kits from Genisphere of Hatfield, Pennsylania, are designed to label miRNAs and other small RNAs with Genisphere's 3DNA dendrimers. If you don't want to do it yourself, companies such as molecular diagnostics specialists Genaco of Huntsville, Alabama, and genetic services company DNAVision of Charleroi, Belgium, offer miRNA expression profiling and quantitation using Luminex xMAP technology. LC Sciences of Houston, Texas and Icoria of Research Triangle Park offer microarray-based miRNA detection covering all miRNAs currently listed in the public miRNA registry.

\section{Differences matter}

If miRNAs are the new kid on the block in genomics, single nucleotide polymorphisms (SNPs) are already big business (see 'Genotyping gets up to speed', opposite). Your DNA is $99.9 \%$ identical to that of another unrelated human, but it is that last $0.1 \%$ that interests researchers. Much of the difference is made up of SNPs, which are sites in DNA that differ by a single base. Groups of SNPs close to one another on a chromosome are called blocks, and are usually inherited together as a haplotype, thus providing a convenient marker for the other genes in the block. The HapMap project, run by the International HapMap Consortium, aims to create a map of these haplotypes and their SNP tags for future research (see 'SNPs and human disease', below).

Using SNP tags, scientists can more efficiently scan an individual's genome for association with phenotypes, such as disease susceptibility, or reactions to drugs or vaccines. Launched in October 2002, the HapMap project hoped to complete the mapping of one million SNP markers by September 2005. When it achieved this goal months ahead of schedule, the consortium announced this February that it will step up its efforts in the second phase to create an improved map that is five times denser than the first draft. This will enable geneticists to zero in on smaller areas of the genome, locating targets more precisely by using more SNP signposts, increasing coverage from one SNP every 3,000 bases (at present) to one every 600 bases.

Vital to phase 2 is Perlegen Sciences of Mountain View, California, which is testing 4.6 million SNPs from public databases for addition to the HapMap. Last September, funded by a grant from the US National Human Genome Research Institute, Perlegen

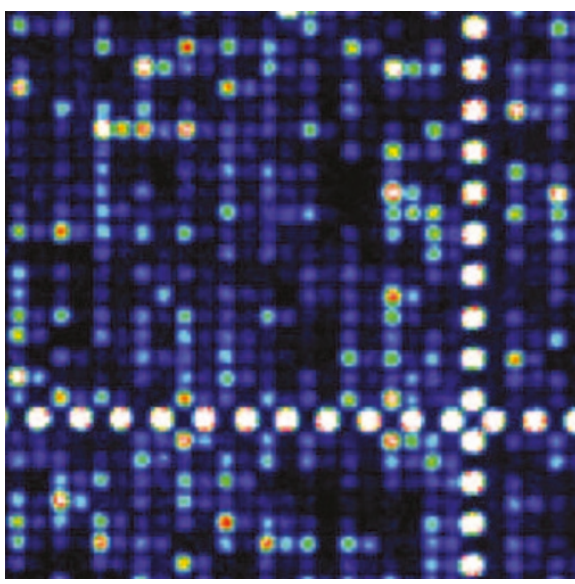

Patterns of life: DNA bound to a small region of an Affymetrix 100K GeneChip Set.

began using high-density oligonucleotide array technology from Affymetrix of Santa Clara, California, to genotype more than 2.25 million unique SNPs from the four HapMap study populations. Perlegen's original goal was to catalogue 600 million genotypes; the new funding in phase 2 should result in more than a billion. The human genome is thought to contain about 10 million SNPs, but not all of these will be useful predictors of disease.

David Cox and his colleagues at Perlegen aim to narrow the field. They have analysed the most common SNPs by mapping 1.5 million SNPs for 71 people from three different ethnic groups: European American, African American and Han Chinese American. The aim is to obtain a high-quality subset of SNPs

\title{
SNPS AND HUMAN DISEASE
}

One goal of the HapMap project is to help reserachers find SNPs associated with human disease. Josephine Hoh at Yale University's School of Public Health and colleagues at Rockefeller University, New York, and the National Eye Institute in Bethesda, have identified an SNP associated with age-related macular degeneration (AMD), a major cause of blindness in people over 60. The SNP is in the gene for complement factor $\mathrm{H}$, leading to a tyrosine to histidine mutation. The researchers studied 96 patients with $A M D$ and 50 healthy controls, and measured the frequency of over 116,000 SNPs in each group. "For the initial screen, we used Affymetrix's set of 100K SNP chips," says team member Robert Klein. "To identify the putative causal mutation, we used PCR to amplify each exon in a number of samples and then resequenced to find all variants in the exons."

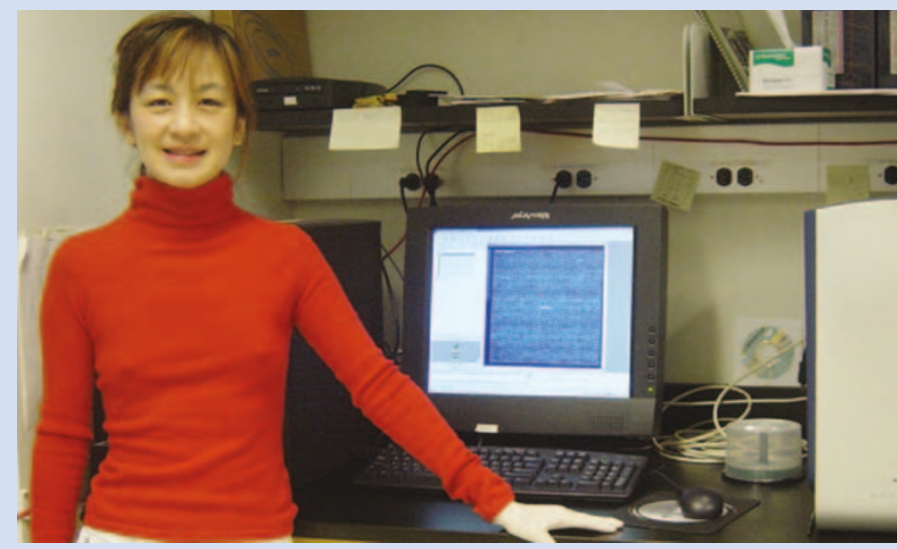

Josephine Hoh uses SNP arrays to find mutations associated with disease.

Hoh and her colleagues found that caucasian patients with AMD are at least four times more likely than usual to have this SNP. How the change causes AMD is not yet known, and one of the next directions for her lab "is to figure out the functional mechanism of complement factor $\mathrm{H}$ in the pathogenesis of AMD", says Hoh.
There are a few clues. The aminoacid change lies in a part of factor $\mathrm{H}$ that interacts with $\mathrm{C}$-reactive protein and heparin, both known to be associated with AMD. And factor $\mathrm{H}$ is known to regulate components of the immune system that are found in drusen, fatty deposits that accumulate in the macula with age. In people with $A M D$, the drusen are larger and more numerous, killing cells needed to nourish adjacent retinal photoreceptors, which eventually results in loss of sight.

SNP mapping is also underway in animal models of human disease. Kent Hunter at the National Cancer Institute ( $\mathrm{NCl})$ in Bethesda, Maryland, uses SNPs to look for cancer-modifying genes in mice. "Ultimately, we hope to identify the particular polymorphic gene or genes that modulate metastatic efficiency," he says. Maxwell Lee at the $\mathrm{NCl}$ is interested in how genetic variation determines gene expression and phenotypes in human cancer and uses SNPs to search for epigenetic markers. "We need to understand more dynamic aspects of the genome including interactions between SNPs and other downstream targets such as chromatin, DNA methylation and gene expression," he says. C.S. 